\title{
Efeitos dos hormônios anabólicos sobre a estrutura, metabolismo e função do músculo esquelético
}

\author{
Effects of anabolic hormones on structural, metabolic \\ and functional aspects of skeletal muscle
}

\author{
Flávio de Oliveira Pires \\ Adriano Eduardo Lima Silva ${ }^{1,2}$ \\ Valmor Tricoli ${ }^{3}$
}

1 Universidade de São Paulo. Departamento de Esporte. Escola de Educação Física e Esporte. Laboratório de Determinantes Energéticos do Desempenho Esportivo. São Paulo, SP. Brasil.

2 Universidade Federal de Alagoas. Centro de Educação. Grupo de Pesquisa em Ciência do Esporte. Maceió, AL. Brasil.

3 Universidade de São Paulo. Departamento de Esporte e Esporte. Laboratório de Adaptações ao Treinamento de Força. São Paulo, SP. Brasil.

Recebido em 17/12/07 Revisado em 02/06/08 Reapresentado em 26/10/08

Aprovado em 21/11/08
Resumo - Este estudo revisou evidências acerca dos efeitos do uso de hormônios anabólicos nos ganhos de força e hipertrofia muscular, abordando os aspectos estruturais, metabólicos e funcionais pelos quais estes hormônios podem potencializar o ganho de força. Atenção especial foi dada ao efeito da dosagem. Foram selecionados estudos por critério de relevância, publicados nos últimos 15 anos, acessados pela base Pubmed. A administração de testosterona (TE) em doses elevadas ( $600 \mathrm{mg} / \mathrm{semana})$ potencializa os efeitos do treinamento de força (TF), promovendo aumento na massa muscular magra, na área de secção transversa das fibras do tipo IIA e IIB, e no número de mionúcleos. Interconversão entre as isoformas da MHC não são evidentes. A interação entre administração de TE e TF também parece modificar algumas vias metabólicas, elevando a síntese protéica, os estoques musculares de ATP-CP e glicogênio, com maior mobilização de gordura. Modificações nas concentrações de 17ß-Estradiol, ou nas relações ACTH-Cortisol e insulina-glucagon, parecem estar envolvidas nestas alterações metabólicas. Com relação ao desempenho físico, a administração de TE pode aumentar os ganhos em força entre 5\% e 20\%, com variações dependentes da dosagem. De outro lado, a ação do hormônio de crescimento $(\mathrm{GH})$ sobre os aspectos estruturais e funcionais é pouco evidente, e seus efeitos recaem mais sobre os aspectos metabólicos. Estudos com maior controle experimental são necessários para a determinação mais ampla dos efeitos dos hormônios anabólicos sobre aspectos estruturais, metabólicos ou funcionais, em humanos.

Palavras-chave: Treinamento de força; Hipertrofia; Testosterona; Esteróides anabólicos androgênios.

Abstract - This study reviewed information regarding the effects of anabolic hormones on strength gain and muscle hypertrophy, emphasizing the physiological mechanisms that may increase muscle strength. Structural, metabolic and functional aspects were analyzed and special attention was paid to the dose-response relationship. The Pubmed database was searched and studies were selected according to relevance and date of publication (last 15 years). The administration of high testosterone doses ( $600 \mathrm{mg} /$ week) potentiates the effects of strength training, increasing lean body mass, muscle fiber type IIA and IIB cross-sectional area, and the number of myonuclei. There is no evidence of conversion between MHC isoforms. The interaction between testosterone administration and strength training seems to modify some metabolic pathways, increasing protein synthesis, glycogen and ATP-CP muscle stores and improving fat mobilization. Changes in $17 \beta$-estradiol concentration or in the ACTH-cortisol and insulin-glucagon ratios seem to be associated with these metabolic alterations. Regarding performance, testosterone administration may improve muscle strength by 5-20\% depending on the dose used. On the other hand, the effects of growth hormone on the structural and functional aspects of skeletal muscle are not evident, with this hormone more affecting metabolic aspects. However, strictly controlled human studies are necessary to establish the extent of the effects of anabolic hormones on structural, metabolic and functional aspects.

Key words: Strength training; Hypertrophy; Testosterone; Androgenic anabolic steroids. 


\section{INTRODUÇÃO}

O treinamento de força (TF) promove alterações estruturais e funcionais que interferem nos aspectos ligados à saúde e ao desempenho esportivo. Dessas, destacam-se o aumento na área de secção transversa (AST) do músculo, a hipertrofia individual das fibras musculares, a conversão da cadeia pesada de miosina (MHC) do subtipo IIB para o IIA, o aumento no ângulo de penação das fibras e o aumento das forças isométrica e dinâmica máximas ${ }^{1}$, ${ }^{2}$. Entretanto, como esses ganhos são limitados em indivíduos treinados como fisiculturistas, levantadores de peso, ou até atletas recreacionais, hormônios esteróides anabólicos androgênicos (EAA), hormônio do crescimento $(\mathrm{GH})$ e/ou insulina são utilizados para potencializar o aumento da força e da massa corporal magra ${ }^{3}$.

Levantamento norte-americano constatou que usuários iniciaram o uso de anabolizantes entre 16 e 30 anos, sendo a maioria $(78,4 \%)$ constituída de atletas não-competitivos ${ }^{4}$. Das substâncias utilizadas estão os EAA como a testosterona (TE), o GH e o fator de crescimento ligado à insulina (IGF-I) $)^{3}$. A TE foi a droga mais utilizada ${ }^{4}$, com doses superiores a $1000 \mathrm{mg} / \mathrm{semana}$. Estes dados são alarmantes devido aos efeitos deletérios e da possibilidade de dependência química e psicológica decorrentes desse uso ${ }^{3}$.

$\mathrm{O}$ aspecto mais preocupante foi a dosagem utilizada pelos usuários ( 1000 mg), bem superior à considerada segura $(\sim 600 \mathrm{mg})$, ou utilizada em tratamentos com reposição hormonal ${ }^{5}$. Essas diferenças têm gerado discordância entre o real efeito dos EAA $^{6}$. Atletas acreditam que os EAA aumentam a força e a AST do músculo, enquanto nem todos os estudos confirmam essa premissa, ${ }^{7,8}$. Esse desacordo tem levado ao uso indiscriminado de EAA.

Problemas no controle da dosagem podem levar a conclusões equivocadas. Por razões éticas, estudos longitudinais são conduzidos com doses consideradas seguras, bem menores do que as empregadas na prática $^{6}$. Estudos transversais tentam amenizar esse problema, mas por razões intrínsecas ao desenho experimental, não controlam aspectos como a influência genética e o tipo de EAA ${ }^{9}$. Mesmo os estudos longitudinais em humanos, frequentemente, apresentam baixo controle experimental, sem a presença de grupo controle com designação cega, ou duplo cega $^{10}$. Outros problemas experimentais envolvem a metodologia para avaliar as alterações morfológicas, metabólicas e no ganho de força muscular.

A presente revisão apresenta evidências acerca dos efeitos do uso de EAA sobre o ganho de força e hipertrofia muscular. Mecanismos fisiológicos pelos quais os EAA potencializam o ganho de força como as alterações estruturais, metabólicas e funcionais, foram abordados. Embora o TF possa diferir em relação à sua natureza, nenhuma distinção foi feita entre os diferentes tipos de força muscular dos estudos abordados, permitindo assim, suposições teóricas mais consistentes.

\section{ALTERAÇÕES MORFOLÓGICAS}

Em geral, o treinamento diminui o tecido gordo e aumenta o tecido magro devido, principalmente, ao aumento no tecido muscular ${ }^{2}$. Não há um consenso quanto aos efeitos do uso de recursos ergogênicos sobre os diversos tecidos, em especial, sobre o aumento da massa muscular ${ }^{7}$. Dentre os fatores envolvidos nesse desacordo estão a dosagem utilizada pela maioria dos estudos, frequentemente, abaixo da utilizada pelos atletas 6 , e o efeito da combinação entre treinamento de força e recurso ergogênico.

Bhasin et al. (2001) e Sinha-Hikim et al. $(2002)^{11}$ demonstraram o efeito isolado da dosagem de TE sobre as adaptações morfológicas. Bhasin et al. (2001) dividiram aleatoriamente 61 indivíduos com função gonodal normal em cinco grupos. Ao longo de 20 semanas, os grupos receberam doses semanais de TE, as quais foram: 1) $25 \mathrm{mg}$; 2) 50 mg; 3) 125 mg; 4) 300 mg; 5) 600 mg. Concomitante ao uso de TE exógena, foi ministrada injeção mensal de um agonista do hormônio de liberação gonadotrópico $(\mathrm{GnRH})$, para suprimir a produção endógena de TE. Foi observado um aumento na massa corporal magra e no volume dos músculos da coxa apenas nos três grupos de maior dosagem, porém com diminuição da massa gorda apenas no grupo que recebeu $600 \mathrm{mg} / \mathrm{semana}$ de TE. Os grupos com menores dosagens aumentaram significantemente a massa gorda. Esse estudo demonstra que o efeito da TE sem a realização de exercícios de força é dependente da dosagem.

Os mesmos pesquisadores demonstraram que o aumento da massa magra é decorrente, ao menos em parte, pelo aumento da AST da fibra muscular ${ }^{11}$. Utilizando metodologia similar à descrita anteriormente, observou-se que dosagens $\geq 50 \mathrm{mg} / \mathrm{semana}$ (50, 300 e $600 \mathrm{mg} / \mathrm{semana})$ de TE aumentavam a AST da fibra do tipo I. Contudo, o aumento na AST da fibra do tipo II foi restrito às dosagens $\geq$ $600 \mathrm{mg} / \mathrm{semana}$. Interessante que a distribuição dos subtipos da MHC não se modificou em quaisquer dosagens, demonstrando que somente o TF poderia alterar o continuum de fibras no sentido 
MHC IIB $\bigoplus$ MHC IIA. Outro ponto relevante é que as dosagens que aumentaram a AST das fibras também aumentaram o número de mionúcleos, provavelmente para manter o domínio nuclear da fibra. Esses resultados mostram que altas dosagens de TE exógena ( $600 \mathrm{mg} / \mathrm{semana})$ provocam aumento na massa muscular de homens com função gonodal normal, independentemente do treinamento.

Em outro estudo, Bhasin et al. (1996) ${ }^{12}$ demonstraram o efeito potencializador do exercício de força combinado com o uso de $600 \mathrm{mg} / \mathrm{semana}$ de TE. Quarenta homens foram divididos em grupo controle placebo sem exercício, grupo placebo mais exercício de força, grupo com administração de TE sem exercício, e grupo com administração de TE mais exercício de força. $O$ grupo submetido aos exercícios de força com administração de TE aumentou significantemente a massa corporal total $(\sim 8 \%)$, a massa magra ( $\sim \%)$ e a AST dos músculos tríceps sural ( 14\%) e quadríceps ( 14\%). Esses achados demonstram que o TF associado à TE é mais efetivo para causar alterações morfológicas do que o uso isolado de TE ou TF. Vale ressaltar que esses estudos utilizaram dosagens de TE consideradas seguras ( $600 \mathrm{mg} / \mathrm{semana})$, bem menores do que a utilizada por indivíduos praticantes de TF com objetivos não competitivos ${ }^{4}$.

Estudos transversais utilizando usuários declarados e não usuários de TE também observaram alterações morfológicas mais expressivas nos usuários ${ }^{9}{ }^{13}$. Kadi et al. (1999) ${ }^{9}$ demonstraram que os levantadores de peso que usavam EAA apresentavam maior AST e maior quantidade de mionúcleos do que os não usuários. Recentemente, D'Antona et al. (2006) ${ }^{13}$ encontraram resultados semelhantes, com maiores AST no músculo vasto lateral e nas fibras do tipo IIB e IIA em fisiculturistas usuários de anabolizantes, em comparação aos não usuários. As isoformas da MHC, a força muscular absoluta e específica (corrigida pela AST da fibra), e a velocidade máxima de encurtamento de cada fibra não foram diferentes entre usuários e não usuários, mostrando que a administração de anabolizantes tem como efeito principal o aumento do tamanho individual das fibras musculares.

Um problema dos estudos transversais supracitados é que os indivíduos auto-administraram as drogas e, portanto, a dosagem não foi rigorosamente controlada. Outra limitação é que, pela natureza do desenho experimental, há uma combinação de diversas drogas e dosagens. Hartgens et al. (2002) ${ }^{14}$ verificaram o efeito da utilização isolada de 200 $\mathrm{mg} / \mathrm{semana}$ de nandrolona e de um "coquetel" auto-administrado com dosagem supraterapêutica, num período de oito semanas. Enquanto não houve aumento na AST das fibras do grupo com nandrolona, um aumento médio de 12,6\% (10,8\% para tipo I e $14,6 \%$ para tipo II) foi observado no grupo que auto-administrou o coquetel. Contudo, o desenho metodológico não permitiu concluir se o efeito observado foi devido à combinação de várias drogas ou simplesmente ao efeito da dosagem.

Outro hormônio bastante utilizado como recurso ergogênico é o GH. O mecanismo pelo qual o GH estimula o crescimento da massa muscular não é bem compreendido, mas acredita-se que seja através de um efeito estimulador na liberação de IGF-I. Entretanto, poucas evidências mostram um efeito significante do $\mathrm{GH}$ em indivíduos saudáveis ${ }^{8}$, sendo mais provável que o uso de $\mathrm{GH}$ tenha maior ação sobre o metabolismo de gordura ${ }^{8}$.

Lange et al. (2002) ${ }^{15}$ demonstraram que o $\mathrm{GH}$ isolado ou associado ao TF não tem efeito sobre a AST do quadríceps ou das fibras musculares individuais. Contudo, há redução da massa corporal gorda e aumento da massa livre de gordura em indivíduos idosos. Como não foi observado aumento na AST do músculo, é possível que a maior massa livre de gordura seja decorrente da maior retenção hídrica.

Apesar da maioria dos estudos com administração de GH ter sido realizada em idosos, estudos em indivíduos jovens têm demonstrado que, mesmo quando combinado ao TF, o uso de GH não promove aumento da massa muscular. Yarasheski et al. (1992) $)^{16}$ observaram aumentos maiores na massa livre de gordura, na quantidade de água corporal e na síntese protéica total após TF associado ao GH do que com TF isoladamente. Entretanto, como a síntese protéica no quadríceps e as circunferências de coxa, braço e tronco não foram diferentes entre os tratamentos, sugere-se que o $\mathrm{GH}$ tenha um potente efeito sobre outros tecidos, que não o muscular. De qualquer maneira, vale destacar que esse estudo também foi realizado com dosagens clínicas (40 $\mu \mathrm{g} / \mathrm{kg} / \mathrm{dia})$, não representando a quantidade utilizada por atletas ou praticantes de exercícios de força.

Em resumo, as evidências apresentadas até o momento apontam para um aumento da massa muscular com a combinação do TF e a administração de TE acima de $300 \mathrm{mg} / \mathrm{semana}$. O efeito do GH parece ser menos preponderante, lançando dúvidas da ação deste hormônio sobre o tecido muscular, já que seus efeitos parecem mais presentes no tecido gordo e retenção hídrica. Os efeitos da combinação de diversas drogas e de supradosagens não são total- 
mente conhecidos, principalmente, por restrições éticas na aplicação dos modelos de pesquisa.

\section{ALTERAÇÕES METABÓLICAS}

As alterações metabólicas também não apontam para uma única direção, possivelmente, devido às limitações experimentais mencionadas anteriormente. Em geral, as alterações no metabolismo de glicogênio (muscular e hepático) e de proteínas parecem sugerir um aumento na síntese, enquanto as alterações no metabolismo de gordura indicam um aumento na degradação $0^{17-20}$. As evidências sobre as modificações no metabolismo de ATP-CP são menos consistentes.

\section{Metabolismo de glicogênio}

Evidências em modelo animal demonstram um maior conteúdo de glicogênio muscular, mas não hepático, após tratamento com EAA ${ }^{18,20}$, embora a magnitude destes resultados pareça depender do tipo de treinamento e do tipo de fibra muscular.

Van Breda et al. $(1993)^{18}$ observaram maiores concentrações intramusculares de glicogênio em repouso, e menores quedas devido ao exercício, em grupo de ratas treinadas juntamente à administração de $\sim 85 \mathrm{mg} / \mathrm{kg}$ peso corporal de TE. No entanto, quando o efeito da interação treino versus TE foi analisado em função do tipo de fibra muscular, verificou-se que as alterações sobre as fibras rápidas glicolíticas (músculo digital longo) eram mais dependentes da interação treino-TE do que as fibras lentas oxidativas (músculo sóleo). Nas fibras lentas, o treinamento executado sem aplicação de TE também aumentou o conteúdo de glicogênio muscular.

Estes achados são corroborados pelos resultados de Cunha et al. $(2005)^{20}$. Em amostra composta por ratos wistars, foram observados efeitos positivos da interação TF-TE $(\sim 2,5 \mathrm{mg} / \mathrm{semana}$ de nandrolona) sobre o conteúdo de glicogênio de fibras rápidas glicolíticas (gastrocnêmios). As fibras lentas oxidativas (sóleo) não sofreram efeito significante do tratamento. $\mathrm{O}$ interessante é que os resultados obtidos em fibras lentas contrapõem os achados de Van Breda et al. (1993) ${ }^{18}$, já que não foi observada elevação no conteúdo de glicogênio muscular no grupo de animais tratados somente com o TF.

Enquanto os mecanismos responsáveis pelas alterações nas fibras de contração rápida podem envolver vias de sinalização intracelular relacionadas à atividade de algumas enzimas-chave do metabolismo glicolítico ${ }^{18,21,22}$, as diferenças envolvendo as fibras de contração lenta poderiam estar relacionadas ao tipo de treinamento empregado nos estudos, ou às diferentes dosagens de EAA. O aumento no conteúdo de glicogênio intramuscular após tratamento com derivados de TE deve ser, entre outras, uma adaptação enzimática ao aumento da atividade da glicogênio sintase (GS), e à diminuição da atividade da glicogênio fosforilase (GP) e G-6-P quinase. Enquanto a GS é responsável pela síntese de glicogênio, a GP e a G-6-P quinase são enzimas marcadoras do metabolismo glicolítico. A diferenciação nas respostas destas enzimas não apenas explicaria a maior preservação dos estoques de glicogênio intramuscular ao uso de EAA, mas também as diferenças observadas entre os tipos de fibras, já que estas enzimas possuem atividades distintas dependendo do subtipo de fibra ${ }^{18}$.

Uma outra via de sinalização intracelular também pode estar envolvida. Max e Toop ${ }^{21}$ verificaram em modelo animal, maior transporte de glicose intramuscular em animais manipulados com propionato de TE. Estes resultados são corroborados pelos achados de Holmäng et al. ${ }^{22}$, os quais observaram maior transporte de glicose intramuscular e menor consumo de glicogênio após tratamento com TE. Estes dados também podem ser uma explicação alternativa para as diferenças observadas entre fibras rápidas e lentas, já que os receptores androgênio-específicos apresentam atividades diferentes entre as diferentes fibras ${ }^{21}$.

Tomados juntos, esses resultados podem justificar algumas das alterações morfológicas e funcionais observadas em humanos. Os maiores níveis de força e a maior AST do músculo de atletas treinados em força e usuários de $\mathrm{EAA}^{9,12,23}$, podem ser uma consequência do maior conteúdo de glicogênio muscular após o emprego de EAA associado ao TF. Poucos são os efeitos da manipulação isolada de EAA sobre o conteúdo de glicogênio hepático, pois este parece ser alterado apenas em função do treinamento. Contudo, estas inferências devem ser consideradas com cautela, haja vista as distâncias entre modelos animais e humanos, e entre as doses utilizadas na prática e as utilizadas nos estudos.

\section{Metabolismo de gordura}

As alterações no metabolismo de gorduras estão aparentemente ligadas a sinalizadores intracelulares que são ativados quando da ação da TE. Parece haver um incremento na mobilização de gordura após a utilização de EAA, indicado, indiretamente, pelo menor percentual de gordura corporal em atletas que administram EAA ${ }^{3,7}$. Esta hipótese ainda é controversa, pois nem todos os estudos demons- 
traram aumento na eficiência do metabolismo de gordura com a utilização de $\mathrm{TE}^{18}$.

Alguns mecanismos poderiam explicar o efeito positivo sobre o metabolismo de gordura. Primeiro, um aumento na oxidação de lipídeos ao uso de EAA poderia resultar da elevação na atividade da palmitol-carnitina-transferase e da citocromo oxidase-C, enzimas marcadoras da $\beta$-oxidação e da cadeia transportadora de elétrons, ou da maior atividade do ciclo de Randle ${ }^{18}$. Outra via que pode explicar o aumento no metabolismo de gordura é a conversão da TE para estradiol (E), ou especificamente para 17 $\beta$-E. Quando doses suprafisiológicas de TE são manipuladas, quantidades relevantes de TE são convertidas a $17 \beta-E^{20}$. O 17 $\beta$-E é conhecido por elevar o metabolismo de lipídeos e preservar o conteúdo de glicogênio muscular e hepático. Kendrick e Ellis (1991)24 observaram maiores oxidação de gordura e preservação dos estoques de glicogênio muscular com o uso de $17 \beta$-E. Embora a ação do $17 \beta$-E seja sítio específico, sugere-se que a manipulação de TE eleve o metabolismo de gordura via conversão à $17 \beta-E^{20,24,25}$.

\section{Metabolismo de proteínas}

Diversos estudos demonstraram significantes incrementos na massa magra após utilização de EAA associado ou não ao TF ${ }^{9}, 10,12$, sugerindo um balanço positivo entre síntese e degradação de proteínas ${ }^{19,}$ 26. Estudos em humanos demonstraram aumentos de até $27 \%$ na síntese protéica após ingestão de TE entre 100 e 200 mg/semana, entre 4 e 12 semanas $^{26-28}$, sem qualquer alteração nos níveis de degradação ${ }^{19}$. $\mathrm{O}$ incremento no balanço protéico positivo após o uso de EAA deve ser consequência direta do aumentado conteúdo nuclear. Neste caso, sugere-se que a elevação na concentração de DNA e RNAm ocorram para manter a razão área de citosol/conteúdo nuclear (domínio nuclear) em níveis ótimos ${ }^{9,26}$.

A liberação de GH é outro mecanismo sugerido como indutor da síntese protéica. Esse hormônio estimula o eixo de secreção do IGF-I, aumentando a liberação de fatores de crescimento acoplados à proteína e estimulando a síntese protéica e a diferenciação das células satélites ${ }^{16,26}$. Assim, tanto TE quanto GH devem ser capazes de aumentar a síntese protéica quando doses suprafisiológicas são utilizadas ${ }^{16,26}$. Consequentemente, a hipertrofia observada poderia ser considerada como uma simples resposta à elevada síntese protéica estimulada pela ação da TE ou $\mathrm{GH}$, com relação à dose-dependente ${ }^{5}$.

\section{Metabolismo de ATP-CP}

Estudos em modelo animal demonstraram um incremento dos estoques de ATP, com reduções na atividade da creatina quinase $(\mathrm{CK})$ em repouso ou durante exercício, após manipulação de doses de EAA entre 1,25 e 3,75 mg/kg de massa corporal ${ }^{27}$, 29. Estas respostas também foram observadas em humanos, com menores níveis sanguíneos de CK em usuários de EAA, após exercícios de força com cargas elevadas ${ }^{30}$.

Os mecanismos responsáveis por tais modificações ainda não são bem identificados, embora sejam sugeridos efeitos diretos e indiretos da TE sobre o metabolismo de ATP-CP. Há evidências de que a TE eleve a atividade da enzima mioquinase, aumentando a ressíntese de ATP via ADP na seguinte reação: $\mathrm{ADP}+\mathrm{ADP}_{\otimes} \mathrm{ATP}+\mathrm{AMP}^{29}$. Esta seria a primeira justificativa para a maior concentração de ATP, e possivelmente, $\mathrm{CP}$, em animais tratados com $\mathrm{TE}^{31}$. Essas respostas também devem estar, indiretamente, associadas à maior síntese e menor degradação de proteínas após o emprego de EAA, embora esta última sugestão não seja tão sustentada ${ }^{19,28}$. Assim, as reduções nos níveis e atividade da CK poderiam ser resultantes, ou de uma diminuição no nível de lesão muscular, ou apenas de uma maior estabilidade da membrana celular $^{19,27,29}$, não refletindo alterações metabólicas significativas sobre os estoques de ATP-CP.

\section{ALTERAÇÕES HORMONAIS}

A manipulação de doses suprafisiológicas de hormônios anabólicos também promove modificações sobre o padrão hormonal. Embora a sugestão de relação causal seja problemática, algumas alterações hormonais ocorrem sistematicamente junto às alterações metabólicas.

Há uma forte ligação entre o aumento das concentrações de TE e a supressão do eixo hormônio adrenocorticóide-cortisol (ACTH-cortisol), explicando, parcialmente, a elevada síntese protéica e maior preservação dos estoques de glicogênio ${ }^{18,19}$. Alén e Häkkinen (1985) ${ }^{31}$ observaram redução nos níveis de ACTH durante a manipulação de TE em atletas de levantamento de peso, embora o cortisol não tenha se alterado. A supressão do eixo ACTH-cortisol ocorre, pois há uma ação concorrente entre TE e cortisol para os mesmos receptores de membrana ${ }^{18}$, atenuando os efeitos catabólicos promovidos pelo cortisol.

Concomitantemente, a elevação de TE e a subseqente elevação de $17 \beta$-Estradiol parece 
produzir aumento na relação insulina-glucagon, promovendo elevação da síntese de glicogênio e da degradação de lipídeos ${ }^{17}$. Contudo, em longo prazo, a alteração desta relação pode causar efeitos negativos, gerando aumento da resistência à insulina ${ }^{22}$. Respostas similares em relação à elevação das concentrações de insulina são observadas quando da utilização de $\mathrm{GH}^{16}$.

\section{ALTERAÇÕES NO DESEMPENHO DA FORÇA MUSCULAR}

A hipertrofia e o ganho de força muscular, decorrentes do TF, parecem estar mais associados à intensidade do exercício do que aos outros componentes da periodização ${ }^{32,33}$. $\mathrm{O}$ aumento da força muscular ocorre por um mecanismo quantitativo, baseado nas mudanças no volume muscular e no tamanho de cada fibra, tanto quanto num mecanismo qualitativo, baseado na mudança do continuum das fibras ${ }^{13}$. Estudos longitudinais mostraram incrementos entre $~ 18 \%$ e $38 \%$ na força máxima após TF entre 4 e 12 semanas ${ }^{2}$, 34 . Estas alterações ocorreram em paralelo às alterações estruturais como o aumento na AST, o comprimento fascicular, o ângulo de penação do músculo ${ }^{2}$ e a conversão de MHC IIB para MHC IIA ${ }^{34}$.

Há evidências de que a administração de TE potencialize os ganhos em força, com alterações semelhantes na estrutura muscular como AST e ângulo de penação ${ }^{10}$. Sugere-se que a administração de EAA aumente o tamanho de cada fibra muscular e o número de fibras, embora esta última ainda seja controversa ${ }^{9}$. Ambos dependeriam da ativação de células satélites, os quais podem ser estimulados pela ação dos EAA sobre os receptores androgênios $^{6,35}$. Vale ressaltar que o aumento no número de fibras não foi sistematicamente demonstrado em humanos, embora a maioria dos estudos confirme o aumento do tamanho das fibras musculares justificada pela elevada síntese protéica causada por EAA $^{6,10,13}$.

Como destacado, a TE pode potencializar o aumento da massa muscular promovido pelo TF. Em estudo longitudinal, demonstraram-se maiores mudanças estruturais no músculo esquelético, com maiores ganhos na força dinâmica máxima em sujeitos que combinaram a administração de 600 $\mathrm{mg} / \mathrm{semana}$ de TE ao $\mathrm{TF}^{12}$. Os ganhos de força para o grupo que combinou TE ao TF foram 38\% e $22 \%$ para os membros inferiores e superiores, respectivamente, significantemente maior do que quando do uso isolado de TE (19\% e 10\%) ou ape- nas do TF (21\% e 11\%). Blazevich e Giorgi (2001) ${ }^{10}$, analisando sujeitos treinados em força, observaram que $\sim 300 \mathrm{mg} / \mathrm{semana}$ de TE combinada ao TF foi capaz de elevar a força dinâmica máxima em 16\%, enquanto os sujeitos que realizaram somente o TF apresentaram incrementos de apenas 3,5\%.

Por outro lado, estudos transversais nem sempre demonstram efeito positivo do uso de TE sobre os ganhos em força9, 13 , já que apesar do maior grau de hipertrofia muscular e alterações estruturais nos usuários de TE, nem sempre a força difere significantemente entre atletas usuários e não usuários. Novamente, problemas metodológicos como aspectos genéticos, combinação, duração e dosagem de EAA utilizados, podem limitar as conclusões já que mesmo as doses inferiores às habitualmente usadas por atletas podem potencializar os ganhos em força em $~ 5 \%$ a $20 \%{ }^{36}$.

Com relação ao $\mathrm{GH}$, Blackman et al. (2002) ${ }^{37}$ verificaram o efeito isolado e combinado do $\mathrm{GH}$ e esteróides (ES - TE para os homens e estradiol para as mulheres), sem a realização de exercícios. Nesse estudo, os sujeitos avaliados foram divididos em grupo GH: rhGH e placebo; grupo ES: ES e placebo; grupo placebo: $\mathrm{GH}$ placebo e ES placebo; grupo GH+ES: rhGH + ES. Foi observado que o aumento da força foi maior nos homens, em especial no grupo $\mathrm{GH}+\mathrm{ES}(\sim 6,8 \%)$. As mulheres tiveram aumento na força nos grupos GH+ES e ES, ainda que de maneira não significante.

Outros estudos apresentaram resultados contrários. Lange et al. (2002) ${ }^{15}$ não notaram qualquer efeito adicional no ganho de força em homens que receberam GH combinado ao TF. Embora a isoforma MHC IIB tenha aumentado no grupo que recebeu $\mathrm{GH}$ sem TF, não houve aumentos na força desse grupo. Por outro lado, o grupo que treinou e recebeu $\mathrm{GH}$ apresentou decréscimo na quantidade de isoforma MHC IIB. Seria possível supor uma sobreposição entre as alterações estruturais causadas pela execução exclusiva do TF e as alterações promovidas pelo uso exclusivo de GH. Neste caso, os efeitos inconsistentes sobre o ganho de força muscular quando do uso do GH podem estar associados ao aumento da produção de EAA induzido pelo próprio $\mathrm{TF}^{38}$. Resultados de Yarasheski et al. $(1992)^{16}$ com indivíduos mais jovens corroboram esta sugestão, já que não foi observada nenhuma interação GH-TF sobre as forças dinâmica máxima, isométrica ou de resistência, em 12 semanas.

Tomadas juntas, as evidências apontam para um efeito positivo na combinação de TF e TE sobre o ganho de força muscular. De outro lado, o 
efeito do GH sobre o ganho de força não é consistente, sendo provável que este agente tenha ação mais relevante sobre o aumento da massa corporal magra, diminuição do tecido adiposo e retenção hídrica ${ }^{15,37}$.

\section{CONCLUSÕES}

As inferências apresentadas a partir dos estudos citados permitem a sugestão de algumas respostas ao uso de EAA: alterações morfológicas: aumento da massa magra e da AST do músculo, hipertrofia seletiva de fibras IIA e IIB, elevação do número de mionúcleos; alterações metabólicas: elevação dos estoques de ATP-CP e glicogênio muscular, maior mobilização de gorduras e aumento da síntese protéica; alterações no desempenho em força: aumentos na força dinâmica máxima. Ainda assim, estudos com maior controle experimental, manipulando doses mais próximas da dosagem empregada nas situações reais e utilizando grupos controle, são necessários para a determinação mais ampla dos efeitos dos EAA sobre variáveis morfológicas, metabólicas ou funcionais, em humanos.

\section{Agradecimentos}

O autor Flávio de Oliveira Pires recebe bolsa doutorado CAPES-demanda social.

\section{REFERENCIAS BIBLIOGRÁFICAS}

1. Staron RS, Karapondo DL, Kraemer WJ, Fry AC, Gordon SE, Falkel JE, et al. Skeletal muscle adaptations during early phase of heavy-resistance training in men and women. J Appl Physiol 1994;76(3):1247-1255.

2. Seynnes OR, de Boer M, Narici MV. Early skeletal muscle hypertrophy and architectural changes in response to high-intensity resistance training. J Appl Physiol 2007;102(1):368-373.

3. Hartgens F, Kuipers H. Effects of androgenic-anabolic steroids in athletes. Sports Med 2004;38(4):513-554.

4. Parkinson AB, Evans NA. Anabolic androgenic steroids: a survey of 500 users. Med Sci Sports Exerc 2006;38(4):644-651.

5. Bhasin S, Woodhouse L, Casaburi R, Singh AB, Bhasin $\mathrm{D}$, Berman N, et al. Testosterone dose-response relationships in healthy young men. Am J Physiol Endocrinol Metab 2001;281(6):E1172-E1181.

6. Kuhn CM. Anabolic steroids. Recent Prog Horm Res 2002;57:411-434

7. Kutscher EC, Lund BC, Perry PJ. Anabolic steroids: a review for the clinician. Sports Med 2002;32(5):285296.

8. Rennie MJ. Claims for the anabolic effects of growth hormone: a case of the emperor's new clothes? $\mathrm{Br} \mathrm{J}$
Sports Med 2003;37(2):100-105.

9. Kadi F, Eriksson A, Holmner S, Thornell LE. Effects of anabolic steroids on the muscle cells of strengthtrained athletes. Med Sci Sports Exerc 1999;31(11): 1528-1534.

10. Blazevich A, Giorgi A. Effect of testosterone administration and weight training on muscle architecture. Med Sci Sports Exerc 2001;33(10):1688-1693.

11. Sinha-Hikim I, Artaza J, Woodhouse L, GonzalezCadavid N, Singh AB, Lee MI, et al. Testosteroneinduced increase in muscle size in healthy young men is associated with muscle fiber hypertrophy. Am J Physiol Endocrinol Metab 2002; 283(1):E154-E164.

12. Bhasin S, Storer TW, Berman N, Callegari C, Clevenger B, Phillips J, et al. The effects of supraphysiologic doses of testosterone on muscle size and strength in normal men. N Engl J Med 1996;335(1):1-7.

13. D'Antona G, Lanfranconi F, Pellegrino MA, Brocca L, Adami R, Rossi R, et al. Skeletal muscle hypertrophy and structure and function of skeletal muscle fibres in male body builders. J Physiol 2006;570(Pt 3):611-627.

14. Hartgens F, van Straaten H, Fideldij S, Rietjens G, Keizer HA, Kuipers H. Misuse of androgenic-anabolic steroids and human deltoid muscle fibers: differences between polydrug regimens and single drug administration. Eur J Appl Physiol 2002;86(3): 233-239.

15. Lange KH, Andersen JL, Beyer N, Isaksson F, Larsson $\mathrm{B}$, Rasmussen $\mathrm{MH}$, et al. $\mathrm{GH}$ administration changes myosin heavy chain isoforms in skeletal muscle but does not augment muscle strength or hypertrophy, either alone or combined with resistance exercise training in healthy elderly men. J Clin Endocrinol Metab 2002; 87(2):513-523.

16. Yarasheski KE, Campbell JA, Smith K, Rennie MJ, Holloszy JO, Bier DM. Effect of growth hormone and resistance exercise on muscle growth in young men. Am J Physiol 1992; 262: E261-E267.

17. Bunt JC. Metabolic actions of estradiol: significance for acute and chronic exercise responses. Med Sci Sports Exerc 1990; 22(3): 286-290.

18. Van Breda E, Keizer HA, Geurten P, Van Kranenburg G, Menheere P, Kuipers H, et al. Modulation of glycogen metabolism of rat skeletal muscles by endurance training and testosterone treatment. Eur J Appl Physiol 1993; 424(3-4): 294-300.

19. Ferrando AA, Tipton KD, Doyle D, Phillips SM, Cortiella J, Wolfe RR. Testosterone injection stimulates net protein synthesis but not tissue amino acid transport. Am J Physiol 1998;275(38):E864-E871.

20. Cunha TS, Tanno AP, Moura MJCS, Marcondes FK. Influence of high-intensity exercise training and anabolic androgenic steroid treatment on rat tissue glycogen content. Life Sci 2005;77(9):1030-1043.

21. Max S, Toop J. Androgen enhance in vivo 2-deoxyglucose uptake by rat striated muscle. Endocrinology 1983;113(1):119-126.

22. Holmäng A, Svedberg J, Jennische E, Björntorp P. Effects of testosterone on muscle insulin sensitivity and morphology in female rats. Am J Physiol 1990: 
259(22):E555-E560.

23. Jürimãe J, Albernethy PJ, Quigley BM, Blake K, McEniery MT. Differences in muscle contractile characteristics among bodybuilders, endurance trainers and control subjects. Eur J Appl Physiol 1997;75(4):357-362.

24. Kendrick Z, Ellis GS. Effec of estradiol on tissue glycogen metabolism and lipid availability in exercised male rats. J Appl Physiol 1991;71(5):1694-1699.

25. Braun B, Gerson L, Hagobain T, Grow D, Chipkin SR. No effect short-term testosterone manipulation on exercise substrate metabolism in men. J Appl Physiol 2005;99(5):1930-1937.

26. Urban R.J, Bodenburg YH, Gilkison C, Foxworth J, Coggan AR, Wolfe RR, Ferixando AA. Testosterone administration to elderly men increases skeletal muscle strength and protein synthesis. Am J Physiol Endocrinol Metab 1995;32: E820-E826.

27. Tamaki T, Uchiyama S, Uchiyama Y, Akatsuka A, Roy RR, Edgerton VR. Anabolic steroids increase exercise tolerance. Am J Physiol Endocrinol Metab 2001; 280(6):E973-E981.

28. Sheffield-Moore M, Paddon-Jones D, Casperson SL, Gilkison C, Volpi E, Wolf SE, et al. Androgen therapy induces muscle protein anabolism in older women. J Clin Endocrinol Metab 2006;91(10):3844-3849.

29. Ramamani A, Aruldhas MM, Govindarajulu P. Impact of testosterone and oestradiol on region specificity of skeletal muscle-ATP, creatine phosphokinase and myokinase in male and female wistars rats. Acta Physiol Scand 1999;166(2):91-97.

30. Boone JB, Lambert CP, Flynn MG, Michaud TJ, Rodriguez-Zayas JA, Andres FF. Resistance exercise effects on plasma cortisol, testosterone and creatine kinase activity in anabolic-androgen steroids. Int J Sports Med 1990;11(4):293-297.

31. Alén, M; Häkkinen, K. Physical health and fitness of an elite bodybuilder during 1 year of self-administration of testosterone and anabolic steroids: a case study. Int J Sports Med 1985;6(1):24-29.

32. Goto K, Ishii N, Kizuka T, Takamatsu K. The impact of metabolic stress on hormonal responses and muscular adaptations. Med Sci Sports Exerc 2005;37(6):955-963.

33. Izquierdo M, Ibânez J, Gonzâlez-Badillo JJ, Ratamess NA, Kraemer WJ, Häkkinen K, et al. Detraining and tapering effects on hormonal responses and strength performance. J Strength Cond Res 2007;21(3):768-775.

34. Andersen LL, Andersen JL, Magnusson SP, Suetta C, Madsen JL, Christenssen LR, et al. Changes in human muscle force-velocity relationship in response to resistance training an subsequent detraining. J Appl Physiol 2005; 99(1): 87-94.

35. Erikson A, Lindström M, Carlsson L, Thornell L. Hypertrophic muscle fibers with fissures in power-lifters; fiber splitting or defect regeneration? Histochem Cell Biol 2006; 126(4):409-417.

36. Savulescu J, Foody B, Clayton M. Why we should allow performance enhancing drugs in sport. Br J Sport Med 2004,38(6):666-670.

37. Blackman MR, Sorkin JD, Münzer T, Bellantoni MF, Whitehead JB, Stevens TE, et al. Growth hormone and sex steroid administration in healthy aged women and men. JAMA 2002; 288(18):2282-2291.

38. Sallinen J, Pakarinen A, Ahtiainen J, Kraemer WJ, Volek JS, Häkkinen K. Relationship between diet and serum anabolic hormone responses to heavy-resistance exercise in men. Int J Sports Med 2004;25(8):627-633.

\author{
Endereço para correspondência \\ Flávio de Oliveira Pires \\ Av. Prof. Mello Moraes, 65. \\ Cidade Universitária, Butantã \\ CEP: 05508-030 - São Paulo (SP) - Brasil. \\ E-mail: piresfo@usp.br
}

